\title{
ON RIGIDITY OF AFFINE SURFACES
}

\author{
BARBARA OPOZDA \\ (Communicated by Christopher Croke)
}

\begin{abstract}
Rigidity of nondegenerate Blaschke surfaces in $\mathbf{R}^{3}$ is studied. The rigidity criteria are given in terms of $\nabla R$, where $R$ is the curvature of the Blaschke connection $\nabla$. If the rank of $\nabla R$ is 2 , then the surface is rigid. If $\nabla R=0$, it is nonrigid. In the case where the rank of $\nabla R$ is 1 there are both rigid and nonrigid surfaces. This case is discussed for various types of surfaces.
\end{abstract}

In this paper we study the rigidity problem for nondegenerate Blaschke surfaces. In a short note [C] in 1924, E. Cartan wrote that in general such surfaces are rigid and indicated three classes of exceptions. Some clarifications were offered later in Ślebodziński's paper [Sl]. We propose a definition of rigidity at a point that fits well the fundamental theorems known in affine differential geometry. Applying this method to a large class of surfaces we obtain some rigidity theorems and examples of nonrigid surfaces. The results in this paper are of local nature. Examples of global rigidity theorems are those of Cohn-Vossen type, see [S] and [NO].

The author is grateful to Professor Katsumi Nomizu for calling her attention to the papers of Cartan and Ślebodziński and for valuable comments on this work.

1

Let $\nabla$ be a torsion-free connection on a 2-dimensional manifold $M$ and $f$ : $(M, \nabla) \rightarrow \mathbf{R}^{3}$ be an affine immersion, that is, around each point of $M$ there is a transversal vector field $\xi$ inducing $\nabla$ according to the Gauss formula:

$$
D_{X}\left(f_{*} Y\right)=f_{*}\left(\nabla_{X} Y\right)+h(X, Y) \xi,
$$

where $D$ denotes the standard connection on $\mathbf{R}^{3}$. An immersion is called nondegenerate if the second fundamental form $h$ is nondegenerate at every point of $M$. The Ricci tensor Ric of $\nabla$ is symmetric if and only if $\xi$ can be chosen equiaffine, i.e. with the property that $D \xi$ is tangential. In this case $f$ is called equiaffine and the Weingarten formula can be written as follows

$$
D_{X} \xi=-f_{*} S X
$$

Received by the editors May 31, 1994.

1991 Mathematics Subject Classification. Primary 53A15; Secondary 53B05.

Key words and phrases. Blaschke surface, metric compatible with connection.

The research was supported by the Kambara Fund of Kobe University and the KBN grant 2P30103004.

(C)1996 American Mathematical Society 
A nondegenerate affine immersion $f:(M, \nabla) \rightarrow \mathbf{R}^{3}$ is called Blaschke if Ric is symmetric and for any equiaffine transversal vector field $\xi$ inducing $\nabla$ and $h$ we have $\nabla \theta_{h}=0$, where $\theta_{h}$ is the volume element determined by $h$. We shall also use the name "a Blaschke surface". The parallelism of $\theta_{h}$ is equivalent to the condition $\operatorname{tr}_{h} \nabla h(X, \cdot, \cdot)=0$ and is called the apolarity condition. If an equiaffine immersion is given, then it will be automatically equipped with an equiaffine transversal vector field (defined around a point under consideration) inducing the given connection and $h, S$ will be the second fundamental form and the shape operator induced by the transversal vector field. Equiaffine transversal vector fields inducing the same connection are equal to each other up to a constant (on a connected common domain). The induced second fundamental forms as well as shape operators induced by proportional equiaffine transversal vector fields are proportional. Our study will not depend on a choice of an equiaffine transversal vector field. For an equiaffine immersion the fundamental equations are the following:

$$
\begin{gathered}
R(X, Y) Z=h(Y, Z) S X-h(X, Z) S Y \quad-\text { Gauss, } \\
\nabla h(X, Y, Z)=\nabla h(Y, X, Z) \quad-\text { Codazzi I }, \\
\nabla S(X, Y)=\nabla S(Y, X) \quad-\text { Codazzi II, } \\
h(S X, Y)=h(X, S Y) \quad-\text { Ricci, }
\end{gathered}
$$

where $R$ is the curvature tensor of $\nabla$. For a more detailed exposition of the theory of affine immersions we refer to [NS].

In what follows by a neighbourhood we shall mean a connected open neighbourhood, and the expression "around $x$ " will stand for "in some neighbourhood of $x$ ". All the connections considered in this paper are assumed to be torsion-free. If $K$ is a tensor field of type $(r, k)$, then $\nabla K\left(X_{0}, X_{1}, \ldots, X_{k}\right)=\left(\nabla_{X_{0}} K\right)\left(X_{1}, \ldots, X_{k}\right)$.

Let $f:(M, \nabla) \rightarrow \mathbf{R}^{3}$ be a Blaschke immersion. It is called globally rigid if for every Blaschke immersion $\bar{f}:(M, \nabla) \rightarrow \mathbf{R}^{3}$ the immersions $f$ and $\bar{f}$ are affine equivalent, i.e. $\bar{f}=B f$ for some $B$ from the general affine group $A\left(\mathbf{R}^{3}\right)$. We shall say that $f$ is rigid at $x \in M$ if the following condition is satisfied: If $\bar{f}:\left(U, \nabla_{\mid U}\right) \rightarrow \mathbf{R}^{3}$ is a Blaschke immersion where $U$ is some neighbourhood of $x$, then there is a neighbourhood $V$ of $x, V \subset U$, such that $\bar{f}_{\mid V}=B f_{\mid V}$ for some $B \in A\left(\mathbf{R}^{3}\right)$. An immersion will be called nonrigid at $x$ if it is not rigid at $x$.

Assume now that $\nabla$ is a connection on a 2-dimensional manifold $M$. We define the rank of $\nabla R$ at $x$, and denote it by $r k(\nabla R)_{x}$, as the dimension of the space

$$
i m(\nabla R)_{x}=\operatorname{span}\left\{\left(\nabla_{Z} R\right)(X, Y) Z ; X, Y, Z \in T_{x} M\right\} .
$$

If $\nabla$ and vectors $X, Y \in T_{x} M$ are given, then we set

$$
\begin{aligned}
& \Re_{1}(X, Y)=\left(\nabla_{X} R\right)(X, Y) X, \\
& \Re_{2}(X, Y)=\left(\nabla_{X} R\right)(X, Y) Y+\left(\nabla_{Y} R\right)(X, Y) X, \\
& \Re_{3}(X, Y)=\left(\nabla_{Y} R\right)(X, Y) Y .
\end{aligned}
$$


We have

$$
\begin{aligned}
\Re_{1}(X, Y)= & \nabla \operatorname{Ric}(X, Y, X) X-\nabla \operatorname{Ric}(X, X, X) Y, \\
\Re_{2}(X, Y)= & {[\nabla \operatorname{Ric}(X, Y, Y)+\nabla \operatorname{Ric}(Y, Y, X)] X } \\
& -[\nabla \operatorname{Ric}(X, X, Y)+\nabla \operatorname{Ric}(Y, X, X)] Y, \\
\Re_{3}(X, Y)= & \operatorname{Ric}(Y, Y, Y) X-\nabla \operatorname{Ric}(Y, X, Y) Y .
\end{aligned}
$$

If $f:(M, \nabla) \rightarrow \mathbf{R}^{3}$ is an equiaffine immersion, then

$$
\begin{aligned}
\Re_{1}(X, Y) & =\nabla h(X, Y, X) S X-\nabla h(X, X, X) S Y \\
& +h(Y, X) \nabla S(X, X)-h(X, X) \nabla S(X, Y), \\
\Re_{2}(X, Y) & =2 \nabla h(X, Y, Y) S X-2 \nabla h(Y, X, X) S Y \\
& +h(Y, Y) \nabla S(X, X)-h(X, X) \nabla S(Y, Y), \\
\Re_{3}(X, Y) & =\nabla h(Y, Y, Y) S X-\nabla h(Y, X, Y) S X \\
& +h(Y, Y) \nabla S(Y, X)-h(X, Y) \nabla S(Y, Y) .
\end{aligned}
$$

If $X, Y \in T_{x} M$ are linearly independent, then

$$
r k(\nabla R)_{x}=\operatorname{dim} \operatorname{span}\left\{\Re_{1}(X, Y), \Re_{2}(X, Y), \Re_{3}(X, Y)\right\} .
$$

Througout the paper by a metric compatible with a Ricci-symmetric connection $\nabla$ we shall mean a nondegenerate symmetric form $h$ such that (1.4) is satisfied, $\nabla \theta_{h}=0$ and

$$
\operatorname{tr}_{h} \nabla \cdot R(X, Y) \cdot=0
$$

for every $X, Y$. The last equality is equivalent to: $\operatorname{tr}_{h} \nabla \operatorname{Ric}(\cdot, \cdot, X)=0$ for every $X$. We set

$$
P=h(\nabla R i c, \nabla R i c) .
$$

If $f:(M, \nabla) \rightarrow \mathbf{R}^{3}$ is a Blaschke immersion, then the induced second fundamental form is compatible with $\nabla$. Conversely, the fundamental theorem says (see Theorem 1 in [DNV]) that if $h$ is a metric compatible with $\nabla$ on some simply connected region $U$, then there is a unique modulo $A\left(\mathbf{R}^{3}\right)$ Blaschke immersion $f:\left(U, \nabla_{\mid U}\right) \rightarrow \mathbf{R}^{3}$ inducing $h$. Therefore we have

Lemma 2.1. Let $f:(M, \nabla) \rightarrow \mathbf{R}^{3}$ be a Blaschke immersion with a second fundamental form $h$ around $x \in M$. The immersion $f$ is rigid at $x$ if and only if for every metric $\bar{h}$ defined around $x$ and compatible with $\nabla$ the metrics $h$ and $\bar{h}$ are proportional around $x$. It is nonrigid at $x$ if and only if there is a metric $\bar{h}$ defined around $x$ and compatible with $\nabla$ such that $h$ and $\bar{h}$ are not proportional in any neighbourhood of $x$.

If $A: T_{x} M \rightarrow T_{x} M$ is an endomorphism and $X, Y \in T_{x} M$, then

$$
\begin{aligned}
& h(A Y, A Y) \Re_{1}(A X, A Y)-h(A X, A Y) \Re_{2}(A X, A Y)+h(A X, A X) \Re_{3}(A X, A Y) \\
& \quad=\operatorname{det} A^{3}\left\{h(Y, Y) \Re_{1}(X, Y)-h(X, Y) \Re_{2}(X, Y)+h(X, X) \Re_{3}(X, Y)\right\} .
\end{aligned}
$$

Hence the equality

$$
h(Y, Y) \Re_{1}(X, Y)-h(X, Y) \Re_{2}(X, Y)+h(X, X) \Re_{3}(X, Y)=0
$$

does not depend on a choice of a basis $X, Y \in T_{x} M$. Conditions (2.8) and (2.6) are equivalent.

The following result is, in fact, due to Ślebodziński, see [S1]. 
Proposition 2.2. Let $\nabla$ be a connection on a 2-dimensional manifold and $f$ : $(M, \nabla) \rightarrow \mathbf{R}^{3}$ be a Blaschke immersion. If $r k(\nabla R)_{x}=2$, then $f$ is rigid at $x$.

Proof. Let $X, Y$ be a tangent frame around $x$. Any two symmetric bilinear forms satisfying (2.8) must be proportional around $x$ because the rank of the system of vectors $\Re_{1}(X, Y), \Re_{2}(X, Y), \Re_{3}(X, Y)$ is 2 around $x$.

An immediate consequence of this proposition is the following

Corollary 2.3. Proper affine spheres are rigid at points where the Fubini-Pick invariant is nonvanishing.

Proof. For a proper affine sphere, $S=\lambda i d$ for some nonzero constant $\lambda$. Assume that $X, Y$ is a basis of $T_{x} M$ such that $h(X, X)=1, h(X, Y)=0$, and $h(Y, Y)=\epsilon$, where $\epsilon= \pm 1$. Then the Pick invariant $J=\frac{1}{8} h(\nabla h, \nabla h)$ is given by

$$
J=\frac{1}{2}\left(\nabla h(X, X, X)^{2}+\epsilon \nabla h(X, X, Y)^{2}\right) .
$$

Since $\epsilon \Re_{1}(X, Y)+\Re_{3}(X, Y)=0$, we have that $r k \nabla R_{x}=2$ if and only if $\Re_{1}(X, Y)$ and $\Re_{2}(X, Y)$ or $\Re_{3}(X, Y)$ and $\Re_{2}(X, Y)$ are not proportional around $x$. From $(2.4),(2.9)$ and the apolarity condition it is easily seen that it happens if and only if $J_{x} \neq 0$.

According to Proposition 2.2 the only points of nonrigidity might be those where $r k \nabla R$ is less than 2. In most cases $r k \nabla R=2$. If $r k \nabla R=0$ on $M$, then, by the symmetry of the Ricci tensor, we have $\nabla R=0$, i.e. $\nabla$ is locally symmetric. Locally symmetric Blaschke immersions are nonrigid, see $[\mathrm{Sl}],[\mathrm{O}]_{1}$. The condition $r k \nabla R<2$, however, is not sufficient for nonrigidity. This will be explained in the next sections.

Assume that $r k \nabla R=1$ in a neighbourhood $U$ of $x$. Let $\Gamma$ be a nowhere vanishing section of $i m \nabla R$ on $U$. Fix a tangent frame $X_{1}, Y_{1}$ on $U$. Denote by $g_{1}$ a symmetric bilinear form defined by

$$
\begin{aligned}
& g_{1}(X, X) \Gamma=\left(\nabla_{X} R\right)\left(X_{1}, Y_{1}\right) X, \\
& g_{1}(X, Y) \Gamma=\left(\nabla_{X} R\right)\left(X_{1}, Y_{1}\right) Y+\left(\nabla_{Y} R\right)\left(X_{1}, Y_{1}\right) X, \\
& g_{1}(Y, Y) \Gamma=\left(\nabla_{Y} R\right)\left(X_{1}, Y_{1}\right) Y .
\end{aligned}
$$

By choosing another tangent frame we get a form proportional to $g_{1}$. Similarly taking another section $\Gamma^{\prime}$ of $i m \nabla R$ we get a proportional form. In this way $\nabla R$ determines a "conformal" structure, say $g$, possibly degenerate. Assume first that $g$ has constant rank 1 on $U$. Then ker $g$ is a 1-dimensional distribution on $U$. Let $X$ be a nowhere vanishing section of $\operatorname{ker} g$ on $U$ and $Y$ be any tangent vector field linearly independent of $X$ on $U$. Then $\Re_{1}(X, Y)=0, \Re_{2}(X, Y)=0$ and $\Re_{3}(X, Y) \neq 0$. If $f:\left(U, \nabla_{\mid U}\right) \rightarrow \mathbf{R}^{3}$ is a Blaschke immersion, then, by (2.8), we have $h(X, X)=0$. Therefore $f$ is nonconvex and ker $g$ is an asymptotic distribution for $h$. The above consideration can be carried out at a point. Therefore we have 
Lemma 3.1. Let $f:(M, \nabla) \rightarrow \mathbf{R}^{3}$ be a Blaschke immersion and $M$ be connected. If there is $x \in M$ where $r k \nabla R=1$ and $r k g=1$, then $f$ is nonconvex.

Coming back to the assumptions $r k \nabla R=1$ and $r k g=1$ on $U$ we now assume that the distribution $k e r g$ is $\nabla$-totally geodesic. Let $(u, v)$ be a coordinate system such that $\partial_{u}$ spans ker $g$ and $\nabla_{\partial_{u}} \partial_{u}=0$. Since $h\left(\partial_{u}, \partial_{u}\right)=0, f$ is ruled. Conversely, assume that $f$ is a ruled Blaschke immersion (more precisely locally ruled), that is, $f$ is locally of the form $f(u, v)=p(v)+u q(v)$. It is known that the asymptotic distribution given by $\partial_{u}$ is $\nabla$-totally geodesic (even parallel). By a straightforward computation we also obtain that $\Re_{1}\left(\partial_{u}, \partial_{v}\right)=\Re_{2}\left(\partial_{u}, \partial_{v}\right)=0$. Therefore we have the following characterization of ruled surfaces in terms of the induced connection only.

Lemma 3.2. Let $\nabla R \neq 0$ everywhere on $M$ and $f:(M, \nabla) \rightarrow \mathbf{R}^{3}$ be a Blaschke immersion. The immersion is ruled if and only if $r k \nabla R=1, r k g=1$ and ker $g$ is $\nabla$-totally geodesic on $M$.

The following observation was made by Cartan in [C].

Proposition 3.3. Every ruled surface is nonrigid. More precisely, for every ruled Blaschke immersion $f:(M, \nabla) \rightarrow \mathbf{R}^{3}$ and every $x \in M$ there are infinitely many ruled Blaschke immersions of $\nabla$ defined around $x$ that are not equivalent to $f$. The immersions are parametrized by a family of functions of one variable.

Proof. Let $f=p(v)+u q(v)$ around $x$ and $l(v)$ be an arbitrary function nonvanishing at $x$. Define a symmetric bilinear form $\bar{h}$ by

$$
\bar{h}\left(\partial_{u}, Z\right)=h\left(\partial_{u}, Z\right), \bar{h}\left(\partial_{v}, \partial_{v}\right)=h\left(\partial_{v}, \partial_{v}\right)+l
$$

for every $Z$. By a straightforward computation one can verify that $\bar{h}$ is compatible with $\nabla$. The proof is complete.

By Lemma 3.2 we know that if $\nabla R \neq 0$ at $x$ on a ruled surface, then $\nabla$ can be immersed by a Blaschke immersion around $x$ only by ruled immersions. We shall show that there are no immersions except for those obtained in Proposition 3.3. It is worth to point out that if $\nabla$ is a locally symmetric connection whose Ricci tensor has rank 1 on a ruled Blaschke surface, then the connection can be also immersed by nonruled (for instance convex) immersions, see $[\mathrm{O}]_{1}$.

Assume that $f, \bar{f}:(M, \nabla) \rightarrow \mathbf{R}^{3}$ are Blaschke immersions and $r k \nabla R=r k g=1$ on $M$. Let $h, \bar{h}$ be the respective induced second fundamental forms. We know that $f$ and $\bar{f}$ are nonconvex. Let $(u, v)$ be an asymptotic coordinate system for $h$. By the apolarity we have $\nabla h\left(\partial_{u}, \partial_{v}, \partial_{v}\right)=\nabla h\left(\partial_{v}, \partial_{u}, \partial_{u}\right)=0$. Consequently $\nabla_{\partial_{u}} \partial_{v}=0$. We know that one of the asymptotic distributions for $h$ and $\bar{h}$ is given by ker $g$. Let $\partial_{u}$ spans ker $g$. Then $\bar{h}\left(\partial_{u}, \partial_{u}\right)=0$ and consequently $\nabla \bar{h}\left(\partial_{v}, \partial_{u}, \partial_{u}\right)=$ $\nabla h\left(\partial_{v}, \partial_{u}, \partial_{u}\right)$. Since both volume elements $\theta_{h}$ and $\theta_{\bar{h}}$ are $\nabla$-parallel, we can assume that they are equal and consequenly we can assume that $h\left(\partial_{u}, \partial_{v}\right)=\bar{h}\left(\partial_{u}, \partial_{v}\right)$. Set $\nabla_{\partial_{u}} \partial_{u}=k \partial_{u}+r \partial_{v}$ and $\bar{h}\left(\partial_{v}, \partial_{v}\right)=h\left(\partial_{v}, \partial_{v}\right)+l$. Then $\nabla \bar{h}\left(\partial_{u}, \partial_{v}, \partial_{u}\right)=$ $\nabla h\left(\partial_{u}, \partial_{v}, \partial_{u}\right)-l r$. In order that $\nabla \bar{h}$ be symmetric $l r$ must vanish. If ker $g$ is not totally geodesic at $x$, then $r \neq 0$ around $x$ and consequently $l=0$ around $x$. In this case $f$ is rigid at $x$. If ker $g$ is totally geodesic around $x$, i.e. if $f$ is ruled, then we have $\nabla \bar{h}\left(\partial_{u}, \partial_{v}, \partial_{v}\right)=\nabla h\left(\partial_{u}, \partial_{v}, \partial_{v}\right)+l_{u}$ and $\nabla \bar{h}\left(\partial_{v}, \partial_{u}, \partial_{v}\right)=\nabla h\left(\partial_{v}, \partial_{u}, \partial_{v}\right)$. Hence $l$ depends only on $v$ and $\bar{h}$ is as in the proof of Proposition 3.3. As an additional piece of information we have 
Proposition 3.4. Let $f:(M, \nabla) \rightarrow \mathbf{R}^{3}$ be a Blaschke immersion. If $r k \nabla R=1$ and $r k g=1$ around $x$ and ker $g$ is not totally geodesic at $x$, then $f$ is rigid at $x$.

We are now in a position of proving the following theorem:

Theorem 3.5. Let $f:(M, \nabla) \rightarrow \mathbf{R}^{3}$ be a Blaschke immersion. If $\nabla$ is projectively flat and $P_{x} \neq 0$, where $P$ is defined by (2.7), then $f$ is rigid at $x$.

Proof. We shall first prove

Lemma 3.6. Let $\nabla$ be a projectively flat connection on a 2-dimensional manifold. If $r k(\nabla R)_{x}=1$, then $r k g_{x}=1$.

Proof of Lemma 3.6. Since $\nabla$ is projectively flat, $\nabla$ Ric is symmetric in all arguments. Let $X, Y$ be a $g$-orthogonal basis of $T_{x} M$, i.e. $\Re_{2}(X, Y)=0$. By (2.3) we have $\nabla \operatorname{Ric}(X, Y, Y)=\nabla \operatorname{Ric}(Y, X, X)=0$. Since $\Re_{1}(X, Y)$ and $\Re_{3}(X, Y)$ are proportional, we have by (2.3): $\nabla \operatorname{Ric}(X, X, X)=0$ or $\nabla \operatorname{Ric}(Y, Y, Y)=0$. Hence $g(X, X)$ or $g(Y, Y)$ vanishes, which finishes the proof of the lemma.

Assume first that $f$ is convex. Since $P_{x} \neq 0,(\nabla R)_{x} \neq 0$. Hence, by Lemmas 3.6, 3.1, we have $r k \nabla R=2$ at $x$ and we can apply Proposition 2.2. Assume now that $f$ is nonconvex. Let $X, Y$ be a frame around $x$ such that $h(X, X)=h(Y, Y)=0$ and $h(X, Y)=1$. Then, by $(2.8), \Re_{2}(X, Y)=0$. By virtue of $(2.3)$ we have $\nabla \operatorname{Ric}(X, X, Y)=\nabla \operatorname{Ric}(Y, Y, X)=0$. Therefore

$$
P=2 \nabla \operatorname{Ric}(X, X, X) \nabla \operatorname{Ric}(Y, Y, Y) .
$$

If $P_{x} \neq 0$, then, using also (2.3), we see that $\Re_{1}(X, Y)$ and $\Re_{3}(X, Y)$ are linearly independent. Again we can use Proposition 2.2 to finish the proof.

In this section we shall study the case where $r k \nabla R=1, r k g=2$ and $g$ is indefinite on some neighbourhood of a point $x$. There are some important classes of affine immersions of this category. For instance, as an immediate consequence of formula (2.8) and Lemma 3.1 we get

Lemma 4.1. If $f:(M, \nabla) \rightarrow \mathbf{R}^{3}$ is a convex Blaschke immersion and $r k \nabla R=1$ at $x$, then $r k g_{x}=2$ and $g_{x}$ is indefinite.

If $r k \nabla R=1, r k g=2$ and $g$ is indefinite on $M$, then there are two asymptotic distributions for $g$. Around every point of $M$ there is a coordinate system $(u, v)$ such that $\partial_{u}, \partial_{v}$ span the distributions.

In order to treat Blaschke immersions with indefinite $g$ we can use the following

Lemma 4.2. Let $f:(M, \nabla) \rightarrow \mathbf{R}^{3}$ be a Blaschke immersion and $\nabla$ have the properties: $r k \nabla R=1, r k g=1, g$ is indefinite around $x$. Let $X, Y$ be a frame spanning asymptotic distributions for $g$ around $x$. The immersion $f$ is nonrigid at $x$ if and only if there is a nowhere vanishing function $\alpha$ defined on an open neighbourhood $U$ of $x$ such that for every neighbourhood $V \subset U$ of $x$ the function $\alpha_{\mid V}$ is not identically equal to 1 and

$$
\left(X \alpha^{-1}\right) h(Y, Y)=b_{1} h(X, X)\left(\alpha^{-1}-\alpha\right),
$$




$$
(Y \alpha) h(X, X)=a_{2} h(Y, Y)\left(\alpha-\alpha^{-1}\right),
$$

where $a_{2}, b_{1}$ are given by $\nabla_{X} X=a_{1} X+a_{2} Y, \nabla_{Y} Y=b_{1} X+b_{2} Y$. Every such $\alpha$ gives a Blaschke immersion of $\nabla$ nonequivalent to $f$.

Proof. Assume that $\bar{h}$ is a metric compatible with $\nabla$ defined around $x$. We may assume that $\theta_{h}=\theta_{\bar{h}}$. By $(2.8)$ we have $h(X, Y)=\bar{h}(X, Y)=0$. Therefore there is a nowhere vanishing function $\alpha$ such that

$$
\bar{h}(X, X)=\alpha h(X, X), \bar{h}(X, Y)=0, \bar{h}(Y, Y)=\alpha^{-1} h(Y, Y) .
$$

Set $\nabla_{Y} X=d_{1} X+d_{2} Y, \quad \nabla_{X} Y=c_{1} X+c_{2} Y$. By the first Codazzi equation for $h$ we get

$$
\begin{gathered}
X h(Y, Y)-2 c_{2} h(Y, Y)=-\left(d_{2} h(Y, Y)+b_{1} h(X, X)\right), \\
Y h(X, X)-2 d_{1} h(X, X)=-\left(c_{1} h(X, X)+a_{2} h(Y, Y)\right) .
\end{gathered}
$$

From the Codazzi equation for $\bar{h}$ we obtain

$$
\begin{array}{r}
\left(X \alpha^{-1}\right) h(Y, Y)+\alpha^{-1} X h(Y, Y)-2 c_{2} \alpha^{-1} h(Y, Y) \\
=-d_{2} \alpha^{-1} h(Y, Y)-\alpha b_{1} h(X, X), \\
(Y \alpha) h(X, X)+\alpha Y h(X, X)-2 d_{1} \alpha h(X, X) \\
=-c_{1} \alpha h(X, X)-a_{2} \alpha^{-1} h(Y, Y) .
\end{array}
$$

Comparing the last formulas with (4.4), (4.5) we get (4.1) and (4.2). Conversely, if a function $\alpha$ has properties mentioned in the lemma, then defining $\bar{h}$ by (4.3) we get a metric compatible with $\nabla$. The assertion now follows from Lemma 2.1.

We shall apply the above lemma to Blaschke immersions whose affine shape operator $S$ is diagonalizable, has rank 1 and $i m S$ is $\nabla$-parallel. The geometric interpretation of the last two properties is that the equiaffine transversal vector field inducing $S$ is planar, see $[\mathrm{O}]_{2}$. Let us briefly recall the construction of such immersions. Let $(u, v)$ be a coordinate system on $\mathbf{R}^{2}$. Let $U$ be an open subset on which a function $a(u)$ and a positive valued function $\phi(u, v)$ satisfy the equation

$$
\epsilon_{1} \phi_{u u}+\epsilon_{1} \frac{a^{\prime}}{2} \phi_{u}+\epsilon_{2} e^{-a} \phi_{v v}=-\phi
$$

where $\epsilon_{1}, \epsilon_{2}$ are \pm 1 . Let

$$
\begin{aligned}
\nabla_{\partial_{u}} \partial_{u} & =\left((\log \phi)_{u}-\frac{a^{\prime}}{2}\right) \partial_{u} \\
\nabla_{\partial_{u}} \partial_{v} & =(\log \phi)_{v} \partial_{u}, \\
\nabla_{\partial_{v}} \partial_{v} & =-\epsilon_{1} \epsilon_{2}(\log \phi)_{u} e^{a} \partial_{u} .
\end{aligned}
$$


Then $h$ given by

$$
\begin{aligned}
& h\left(\partial_{u}, \partial_{u}\right)=\epsilon_{1} \phi e^{-a}, \\
& h\left(\partial_{u}, \partial_{v}\right)=0, \\
& h\left(\partial_{v}, \partial_{v}\right)=\epsilon_{2} \phi
\end{aligned}
$$

is compatible with $\nabla$, the Blaschke immersion of $\nabla$ inducing $h$ has diagonalizable shape operator $S$ of rank 1 and im $S$ is $\nabla$-parallel. Conversely, on every Blaschke surface whose shape operator $S$ is diagonalizable, has rank 1, and $i m S$ is $\nabla$-parallel there is an atlas of coordinates in which $\nabla$ and $h$ are expressed as above. By a straightforward computation we obtain

$$
\Re_{1}\left(\partial_{u}, \partial_{v}\right)=0, \quad \Re_{3}\left(\partial_{u}, \partial_{v}\right)=0, \quad \Re_{2}\left(\partial_{u}, \partial_{v}\right)=\epsilon_{2} a^{\prime} e^{a} \partial_{u} .
$$

Equations (4.1), (4.2) are given by $\alpha_{v}=0$, i.e. $\alpha$ depends only on $u$, and

$$
\left(\alpha^{-1}\right)^{\prime}=(\log \phi)_{u}\left(\alpha-\alpha^{-1}\right) .
$$

If $(\log \phi)_{u v} \neq 0$ on some neighbourhood $U$ of $x$, then the only solution of (4.12) is $\alpha=1$ on $U$ and consequently $f$ is rigid at $x$. If $(\log \phi)_{u v}=0$ around $x$, then there are infinitely many nonequivalent to $f$ Blaschke immersions of $\nabla$ around $x$. Both situations occur. Namely, let $\phi_{1}(u), \phi_{2}(v)$ be functions satisfying the equations

$$
\begin{gathered}
\epsilon_{2} \phi_{2}^{\prime \prime}+k \phi_{2}=0, \\
\epsilon_{1} e^{a} \phi_{1}^{\prime \prime}+\epsilon_{1} e^{a} \frac{a^{\prime}}{2} \phi_{1}^{\prime}+e^{a} \phi_{1}-k \phi_{1}=0,
\end{gathered}
$$

where $k$ is an arbitrary constant. Then $\phi=\phi_{1} \phi_{2}$ is a solution of (4.8). For such a $\phi$ we have $(\log \phi)_{u v}=0$. For any constant $c \geq 0$ the function

$$
\alpha=\phi_{1}\left(c+\phi_{1}^{2}\right)^{-\frac{1}{2}}
$$

is a solution of (4.12). If $c \neq 0$, then $\alpha \neq 1$ around $x$. On the other hand if $\phi_{1}, \bar{\phi}_{1}$ are R-linearly independent solutions of (4.14) and $\phi_{2}$ and $\bar{\phi}_{2}$ are $\mathbf{R}$-linearly independent solutions of (4.13), then $\phi=\phi_{1} \phi_{2}+\bar{\phi}_{1} \bar{\phi}_{2}$ is a solution of (4.8) such that $(\log \phi)_{u v} \neq 0$ around some point.

In this section we shall continue the study of the rigidity problem for Blaschke surfaces whose shape operator $S$ has constant rank 1. Such surfaces with nondiagonalizable shape operator are nonrigid. Namely we have (see [OS])

Lemma 5.1. If $f:(M, \nabla) \rightarrow \mathbf{R}^{3}$ is an equiaffine immersion whose shape operator is singular and nondiagonalizable everywhere, then $f$ is ruled.

We shall now prove 
Lemma 5.2. Let $f:(M, \nabla) \rightarrow \mathbf{R}^{3}$ be a Blaschke immersion whose shape operator has rank 1 around $x$. If $r k \nabla R=1$ at $x$, then the distribution im $S$ is $\nabla$-parallel at $x$. If im $S$ is $\nabla$-parallel at $x$, then $r k \nabla R<2$ at $x$.

Proof. The second assertion follows from formula (2.4). Namely, if $i m S$ is $\nabla$ parallel at $x$, then $(\nabla S)_{x}$ has values in $i m S$. Consequently, $(\nabla R)_{x}$ has values in im $S$ which is 1-dimensional by assumption. In order to prove the first assertion consider the sets $M_{1}, M_{2}: M_{1}=\left\{x \in M ; S_{x}\right.$ is diagonalizable $\}, M_{2}=M \backslash M_{1}$. The set $M_{1}=\left\{x ; S_{x}^{2} \neq 0\right\}$ is open. Assume first that $x \in M_{1}$. Hence $S$ is diagonalizable around $x$ and the distributions im $S$ and ker $S$ are complementary. By the Ricci equation the distributions are $h$-orthogonal. Let $(u, v)$ be a coordinate system around $x \in M$ such that $\partial_{u}$ spans im $S$ and $\partial_{v}$ spans ker $S$. By the second Codazzi equation we get $\nabla_{\partial_{v}} \partial_{u}=C \partial_{u}$ for some function $C$. Set $\nabla_{\partial_{u}} \partial_{u}=$ $A \partial_{u}+B \partial_{v}$. Suppose that $i m S$ is not $\nabla$-parallel at $x$, i.e. $B \neq 0$ around $x$. By the Gauss equation we have im $S=i m R\left(\partial_{u}, \partial_{v}\right)=\operatorname{ker} R\left(\partial_{u}, \partial_{v}\right)$. Therefore

$$
R\left(\partial_{u}, \partial_{v}\right) \partial_{u}=0, \quad R\left(\partial_{u}, \partial_{v}\right) \partial_{v}=\iota \partial_{u}
$$

for some nowhere vanishing function $\iota$ around $x$. Then

$$
\Re_{1}\left(\partial_{u}, \partial_{v}\right)=-B \iota \partial_{u}
$$

We also have

$$
\Re_{2}\left(\partial_{u}, \partial_{v}\right)=\iota_{u} \partial_{u}+\iota \nabla_{\partial_{u}} \partial_{u}+Z
$$

where $Z \in i m R\left(\partial_{u}, \partial_{v}\right)=i m S$. Using formulas (5.2), (5.3) and taking into account that $B \neq 0, \iota \neq 0$ and $r k \nabla R=1$ we get a contradiction. Hence $i m S$ must be $\nabla$-parallel at $x$.

If $x \in$ int $M_{2}$, then the assertion follows from Lemma 5.1 and properties of ruled surfaces. If $x \notin$ int $M_{2}$ and $x_{n} \in M_{1}$ is a sequence converging to $x$, then by the previous part of the proof we have that im $S$ is $\nabla$-parallel at every $x_{n}$, and consequently at $x$. The proof is complete.

A consequence of the above lemma is the following

Theorem 5.3. If $f:(M, \nabla) \rightarrow \mathbf{R}^{3}$ is a Blaschke immersion, its shape operator $S$ has rank 1 around $x$ and $i m S$ is not $\nabla$-parallel at $x$, then $f$ is rigid at $x$.

Proof. Since $i m S$ is not parallel at $x,(\nabla R)_{x} \neq 0$. By Lemma 5.2 we have $r k \nabla R=$ 2 at $x$. The assertion now follows from Proposition 2.2.

A geometric interpretation of the condition $r k S=1$ is that the transversal vector field $\xi$ inducing $S$ is a curve, i.e. for every $x \in M$ there is a coordinate system $(u, v)$ in which $\xi$ depends on one variable. The parallelism of $i m S$ is equivalent to the fact that $\xi$ is a planar curve. For more details see $[\mathrm{O}]_{2}$, [OS]. Therefore the last result can be reformulated as follows.

Corollary 5.4. If an equiaffine transversal vector field $\xi$ for a Blaschke immersion $f:(M, \nabla) \rightarrow \mathbf{R}^{3}$ is a curve and $\operatorname{det}\left(\xi, \xi^{\prime}, \xi^{\prime \prime}\right) \neq 0$ at $x$, then $f$ is rigid at $x$. 


\section{REFERENCES}

[C] E. Cartan, Sur la connexion affine des surfaces, C. R. Acad. Sci. Paris 178 (1924), 292295.

[DNV] F. Dillen, K. Nomizu, L. Vrancken, Conjugate connections and Radon's theorem in affine differential geometry, Monatsh. Math. 109 (1990), 221-235. MR 91e:53015

[NO] K. Nomizu, B. Opozda, Integral formulas for affine surfaces and rigidity theorems of CohnVossen type, Geometry and Topology of Submanifolds IV, World Scientific, Singapore, 1993, pp. 133-142. MR 93j:53012

[NS] K. Nomizu, T. Sasaki, Affine Differential Geometry (1994), Cambridge University Press. CMP 95:06

$\left[\mathrm{O}_{1}\right]$ B. Opozda, Locally symmetric connections on surfaces, Results in Math. 1481 (1991), 185-191. MR 93b:53014

$\left[\mathrm{O}_{2}\right] \quad$ B. Opozda, A class of projectively flat surfaces, Math. Z. 219 (1995), 77-92. CMP 95:15

[OS] B. Opozda, T. Sasaki, Surfaces whose affine normal images are curves, Kyushu J. Math. 49 (1995), 1-10.

[S] U. Simon, Global uniqueness for ovaloids in Euclidean and affine differential geometry, Tôhoku Math. J. 44 (1992), 327-334. MR 93i:53012

[Sl] W. Ślebodziński, Sur quelques problèmes de la théorie des surfaces de l'espace affine, Prace Mat. Fiz. 46 (1939), 291-345.

Instytut Matematyki, Jagiellonian University, Reymonta 4, 30-059 Kraków, Poland

E-mail address: opozda@im.uj.edu.pl 